Advances in Complex Systems

(C) World Scientific Publishing Company

\title{
Competition and the dynamics of group affiliation
}

\author{
Nicholas Geard and Seth Bullock \\ School of Electronics and Computer Science, University of Southampton \\ Southampton, SO17 1BJ, United Kingdom \\ $\{n l g, s g b\} @ e c s . s o t o n . a c . u k$
}

\begin{abstract}
How can we understand the interaction between the social network topology of a population and the patterns of group affiliation in that population? Each aspect influences the other: social networks provide the conduits via which groups recruit new members, and groups provide the context in which new social ties are formed. Given that the resources of individuals are finite, groups can be considered to compete with one another for the time and energy of their members. Such competition is likely to have an impact on the way in which social structure and group affiliation co-evolve. While many social simulation models exhibit group formation as a part of their behaviour (e.g., opinion clusters or converged cultures), models that explicitly focus on group affiliation are less common. We describe and explore the behaviour of a model in which, distinct from most current models, individual nodes can belong to multiple groups simultaneously. By varying the capacity of individuals to belong to groups, and the costs associated with group membership, we explore the effect of different levels of competition on population structure and group dynamics.
\end{abstract}

Keywords: social networks; group affiliation; simulation

\section{Introduction}

Groups are an important social phenomenon, representing an intermediate level of organisation between individuals and society as a whole. Many types of groups can be identified, centred around professional, recreational, social, political, charitable and other interests. Groups have aims, often related to either furthering the interests of their members in a way that could not be achieved by individual members acting alone. For example, groups may provide their members with company or social status, or provide a united voice arguing for political change, collective bargaining or fund-raising. Some types of groups, such as religious or political groups, may be exclusive, in that membership in one group precludes membership in other groups of that type, while others are non-exclusive: a single individual may be a member of multiple recreational or charitable groups, for example. The pattern of group affiliation across a population changes over time as new groups are formed, individuals join and leave existing groups, and old groups die away.

Groups and social networks share a reflexive relationship. Groups grow via the 
recruitment of new members through existing social contacts [39]. At the same time, groups act as a foci for the formation of new social ties, as group activities bring previously unknown people into contact [13]. The dynamics of groups are therefore an emergent property of decisions made by individuals about both their affiliations and their social contacts. One general constraint on group membership is that affiliation with a group tends to involve the commitment of time and energy to events associated with that group (e.g., meetings, fund-raising, or engaging in social activities) [25]. Therefore, there is a limit on the number of groups with which a single individual can be actively involved. As a result, groups must compete with one another for the time and resources of their potential members [27, 41, 28].

There are several reasons then to be interested in the dynamics of groups. An understanding of group level processes, and how they emerge from and influence the behaviour of individuals, is a critical part of understanding social order. Is it possible to predict or explain the success or failure of groups formed with a particular social or political agenda? How do organisations adapt and change to changing environmental conditions? How can such an understanding inform strategies of groups seeking to survive and thrive in a complex environment? The relevance of group dynamics to current issues ranges from, for example, the emergence of environmental NGOs amidst the changing social and political landscape of China [43] to the recent surge of right wing extremist and militant groups in the US [40].

Despite the presence of group level behaviour in several different categories of social simulation model, there are few models that focus explicitly on how the dynamics of group affiliation and social structure co-evolve. Most existing models that involve group formation correspond to the scenario in which groups are exclusive. In this paper we explore the implications of relaxing this constraint and allowing individuals to belong to multiple groups simultaneously. We first review theoretical background on group affiliation and existing models that involve group level dynamics. We then describe a minimal non-exclusive group (NEG) model of a system in which group affiliation and social structure co-evolve and use it to investigate the effect that varying the intensity of competition has on population structure and group dynamics. Finally, we summarise our observations and discuss future directions in which the NEG model could be developed.

\section{Theoretical basis: sociological perspectives on social groups}

Groups are integral to the structure and functioning of complex societies, facilitating the organisation of individuals with heterogeneous and specialised needs and skills. Groups are similarly important to the individuals within a complex society, providing the context in which they find "work and recreation, rewards and penalties, struggle and mutual aid" [23]. Interest in the dynamics of groups stretches back at least as far as Simmel, who maintained that the association among individuals via social groups constituted the very fabric of society [38]. He proposed that the transition from pre-modern to modern society had been characterised by a transfor- 
mation in the geometry of social structure. Affiliations in pre-modern society were concentric: an individual owed allegiance to family, town and religion in a series of increasingly large social circles. By contrast, modern society is characterised by an increase in choices of affiliation and an unshackling of the bonds between them; each individual stands at the centre of a set of partially overlapping social circles, the unique intersection of which contributes to their self-definition [33]. The pattern of group affiliations across society will therefore be complex and overlapping.

Social groups in modern societies take a broad range of forms, from families and clubs to unions and religions. One common classification made by sociologists is to distinguish between primary and secondary groups. In the former category are small groups in which all members have direct contact with one another, such as families, teams and friendship groups. In contrast, secondary groups are larger and may be geographically dispersed, with more complex internal structure [10, 23]. In contrast to primary groups, in which people can have little choice of affiliation, secondary groups are typically freely joined and left by individuals, with little influence from government or market forces. The members of a secondary group are typically united by their pursuit of a common interest or goal, which may range from their participation in a particular sport through to a campaign to elect a particular politician. When the goal is finite, as in the latter case, groups may succeed or fail in their aims, and understanding the factors that contribute to the success of groups is an important challenge [9].

It has been argued that voluntary, interest-focused groups may be an important factor influencing societal integration [3]. Such groups have the potential to act as bridges across demographic categories by providing opportunities for interaction that would otherwise be absent $[34,35]$. Thus, they can help to reduce societal cleavages that may result in conflict or inequality, bringing together individuals of disparate social backgrounds according to shared interests or goals. However, this view has been challenged by the observation that many voluntary associations are often homogeneous and therefore reinforce existing societal divisions [26]. Individuals associated with groups can often be distinguished by such demographic variables as their sex, age, race, profession and educational background. One cause of this is homophily - the principle that like attracts like. Given a choice, people are widely observed to preferentially interact with others with whom they share similar characteristics [26]. When most network ties are between similar individuals, and most group recruitment occurs via network ties, the result is homogeneous groups. Not only may this homogeneity have a negative impact on societal integration, it may also be harmful to the interests of the groups: research on social movements indicate that a movement's efficacy (it's ability to achieve its aims) is dependent on the diverse skills and resources that its members can call upon. Such skills and resources are likely to be maximised among heterogeneous groups [30].

Understanding the factors that influence an individual's decision to join or leave groups is a challenge that has received considerable attention $[15,24,27,26,36$, 39]. One theme that has emerged is the importance of social context for an indi- 
vidual's choices of group affiliation. While it is conceivable that individuals make independent decisions about their affiliations on the basis of their personal beliefs and values, evidence suggests that an individual's social context plays an important (perhaps dominating) role. Given the strong coupling between social structure and group affiliation, simulation modelling is an appropriate methodology to help better understand the dynamics of this complex relationship. In the following section we review the existing models that come closest to capturing this relationship, before setting out the assumptions that governed the design of our own model.

\section{Existing models: opinions, cultures and groups}

A variety of existing models capture aspects of the group formation process in which we are interested. While group formation per se was not necessarily the primary focus of these models, they each exhibit affiliation dynamics and their behaviour can be described in terms of clusters of individuals united by some shared property. More general reviews of these models may be found in recent review papers [6]. Here, we focus on the manner in which these models capture the interaction between group affiliation and social evolution, and the extent to which they exhibit inter-group competition.

Opinion dynamics: One important class of models studies the emergence of agreement in groups of people holding diverse opinions on some issue [11, 17]. The set of opinions available to an individual may represent a binary choice (e.g., voting yes or no in a referendum), a choice among discrete options (e.g., alignment to one particular religion), or the choice of a value on a continuum (e.g., where a more nuanced range of views are possible). Various rules for opinion change have been proposed, from simple 'voting' or 'majority rules' models, where individuals adopt the opinion of one or more of their neighbours, through to 'bounded confidence' models, in which individuals are represented by both a continuous opinion and an uncertainty level and influence one another according to the extent that their opinion regions overlap.

A primary motivation for opinion dynamics models is to understand the conditions under which a single opinion will prevail and the conditions that will lead to two (or more) opinions co-existing in a population. Opinions are exclusive - an individual may only hold one opinion on a particular topic at a time. Hence, competition between opinions is intrinsic to the model: one opinion's gain of an adherent is another opinion's loss. Coevolutionary opinion dynamics models have also been explored, in which agreement between individuals is used as the basis for social tie creation $[1,18,21,22]$. Such homophilous rewiring, if rapid with respect to the rate of state update, leads to the emergence of disconnected clusters of nodes, each consisting of a single opinion. 
Cultural evolution: The paradigmatic model of cultural evolution was proposed by Axelrod [2]. He began with the challenge of explaining why, if individuals tend to become more similar over time, we are left with any diversity at all. In Axelrod's model, an individual's culture is defined as a vector of discrete-valued traits. Individuals interact if they share sufficient cultural traits and adopt their neighbours' trait values. Over time, interactions between individuals lead to regions of cultural convergence separated by cultural boundaries (i.e., neighbouring sites who share no cultural traits and hence do not interact). Axelrod found that the number of stable cultures varied with the number of cultural features, and the number of possible traits per feature. There is some overlap between models of opinion formation and cultural evolution, the primary distinction being that opinions are typically modelled as scalar variables, while cultures are modelled as vectors of traits. Axelrod's model has also been extended to include structural change, with individuals able to rewire social ties away from culturally dissonant neighbours [7]. In this model, one possible outcome is for the population to organise into disconnected and culturally homogeneous clusters, similar to those observed in coevolutionary opinion dynamics models.

Group affiliation: Several recent papers have considered group affiliation more directly. A series of models have been proposed based on tag-related mechanisms that facilitate the emergence of groups of cooperating individuals [12]. This class of models range from abstract resource-gathering simulations, in which agents with diverse resource-gathering skills form into complementary groups, through to decentralised computing simulations, in which nodes form into cooperative groups that isolate and avoid exploitation by defectors. In these models group affiliation is an implicit concept arising from the patterns of interaction between individual agents.

A more explicit depiction of group affiliation is used in a model of church membership designed to investigate the effectiveness of different membership strategies on church survival [8]. In this model, members allocate their time and income between church and other secular activities according to a utility function. Churches are differentiated by the cost they demand of their followers, and the extent to which they proscribe participation in secular activities. The simulations found that the most liberal churches (proscribing no or few activities) survived longest, but that, for stricter churches, their was no significant relationship between the number of activities proscribed and the consequent reduction in lifespan. While church membership is exclusive in this model, individuals can participate in multiple secular activities, and competition exists among churches, and also between churches and secular activities.

\section{Non-Exclusive Group Model: Assumptions}

Each of the models above involve some notion of groups, whether they be clusters of opinion, regions of homogeneous culture, or actual organisations. However, af- 
filiation differs from both opinion and culture in several pertinent ways: opinion dynamics models typically consider a single issue, about which individuals may express one of two or more opinions (the model described in [19] is a notable exception, looking at interactions between two issues). In reality, individuals belong to multiple groups simultaneously. The trait vector of the cultural models comes closer to reflecting this scenario; however, the inability of an individual to increase or decrease the number of traits they exhibit during a simulation is limiting. Furthermore, the phenomenon of cultural convergence, where a group of individuals come to share identical trait vectors, has no meaningful analogy in the context of group affiliation. In designing the NEG model, we have drawn upon existing models where relevant, but removed the assumption of exclusive group membership.

Two broadly contrasting approaches to modelling social networks can be distinguished [4]. The first, from the perspective of social scientists, emphasises the importance of the individual node, the nuances of its relationships with other nodes and the effect that this has on its actions. The second, from the perspective of physical scientists, emphasises the network as an aggregate, seeking to explain how particular network structures might explain, or be explained by, particular types of interaction between individual nodes. The NEG model falls in the latter class. We do not explicitly model the utility functions that result in individual agents making the decisions they do, but rather assume that, given a particular context, a given action will be performed at a particular rate across the entire population.

A further distinction may be made between models that aim to be predictive and those whose aim is understanding [14]. The NEG model again falls into the latter class; we aim to better understand the interaction between social structure and group dynamics, without attempting to make predictions about a particular system. By translating a set of assumptions (derived from literature) into a simulation model, we are able to explore their consequences. The key assumptions embodied in the NEG model are as follows:

- Individuals can be located in sociodemographic space according to variables such as their age, gender, social class and geographic location [27, 42].

- Individuals wish to join groups; while not modelled explicitly, we assume that groups provide access to resources that would otherwise be unavailable [32]; furthermore, individuals wish to participate in groups to the maximum extent that their available time and energy allow.

- Individuals prefer to associate with others who are similar to themselves, where similarity is defined in terms of distance in sociodemographic space [26].

- Social ties are the primary avenue of group recruitment [24].

- Groups are an important foci for the formation of new social ties between individuals [39]; however, social ties may also form between 'strangers' [20].

In addition, we make several pragmatic assumptions that we recognise as oversimplifications of real systems, but that allow us to achieve a parsimonious model. 
- New groups are initiated stochastically by individuals.

- Groups of individuals have goals that are, in some fashion, dependent on the continuing investment of time and energy by their members; therefore the success of a group will depend on its ability to attract new members and retain existing members.

- From the perspective of an individual, groups are distinguished only by the sociodemographic composition of their members, and are otherwise equivalent and interchangeable with respect to the benefits they provide.

By temporarily setting aside considerations of individual utility and group efficacy, we can focus more clearly on the effect that competition has on the interaction between group dynamics and social structure. The following section translates these assumptions into a simulation model. In the final section of the paper, we discuss some of the limitations that arise from the assumptions made here, and how they could be mitigated in future models, at the end of the paper.

\section{Non-Exclusive Group Model: Description}

We consider a network of $n$ nodes and $m$ undirected edges, representing individuals and the social ties between them. Each node $i$ has a trait vector of dimension $d$, representing that individual's location in social space [42], a list of affiliated groups, and a 'time and energy' (TAE) capacity. Trait values are bounded between zero and one, and are uniformly distributed. The social distance between two individuals is defined as the Euclidean distance between their trait vectors.

Each group has a TAE cost associated with being a member. The number of groups with which a node can be affiliated is limited by its TAE capacity. For example, a node with TAE capacity of two could be simultaneously affiliated with two groups with TAE cost of one, or one group with TAE cost of two. The social distance between an individual $i$ and a group $g$ is defined as the mean social distance between $i$ and the set of $i$ 's neighbours belonging to $g$.

Two classes of process act on the network. The first class affects social structure: edges may be rewired either to nodes sharing a common state, or at random. The second class affects group affiliation: nodes may either initiate a new group, or be recruited to an existing group by one of their network neighbours. During each time-step of a simulation run, one of the four processes below is chosen to occur with probability proportion to the rates indicated:

(1) Group-oriented rewiring (rate $m p)$ - Choose a random edge $(i, j)$; Choose a random node $k$ that has at least one shared group affiliation with $i$, but to which $i$ is not currently connected; Delete the edge $(i, j)$ and add the new edge $(i, k)$.

(2) Group recruitment (rate $m q$ ) - Choose a random edge $(i, j)$; Choose one of $i$ 's groups and attempt to recruit $j$ to that group (if not already a member). If joining the group would cause $j$ to exceed their TAE capacity, $j$ either leaves 
one or more of their current groups, or refuses the recruitment attempt (see below).

(3) Group initiation (rate $n r$ ) - Choose a random node $i$; create a new group and affiliate $i$ with it. If initiating the group causes $i$ to exceed their TAE capacity, $i$ leaves one or more of their current groups (see below).

(4) Random rewiring (rate $m w$ ) - Choose a random edge $(i, j)$; Choose a random node $k$ to which $i$ is not currently connected; Delete the edge $(i, j)$ and add the new edge $(i, k)$.

As mentioned above, groups have a TAE cost associated with membership and an individual node's affiliations are limited by its TAE capacity. A node initiating a new group will always leave existing groups to make time for the new group. In contrast, a node being recruited to a new group will either leave existing groups, or refuse the recruitment attempt, depending on which set of groups it identifies most strongly with (i.e., is closest to in sociodemographic space). When a node $i$ exceeds its TAE capacity, it considers its social distance from each of its current groups and the new group it is to join. It then leaves groups in order of increasing social distance until either sufficient TAE capacity has been made available to join the new group, or the new group has been rejected. Nodes are able to rejoin groups that they have left if they are invited again at some point in the future.

\section{Results}

For the simulations reported in this paper, parameters governing the size and density of the network and the rates of each process were fixed: $\{n=200 ; m=600 ; d=$ $1 ; p=1.0 ; q=0.1 ; r=0.01 ; w=0.01\}$. The effect that varying these parameters has on the behaviour of adaptive networks is analysed in greater detail elsewhere [5]. To begin, we explore the scenario where all memberships are exclusive (i.e., where each individual can only belong to a single group at a time), demonstrating that the NEG model exhibits comparable behaviour to coevolutionary models of opinions and culture. We then report three further sets of simulations: the first investigates the effect of increasing the capacity of nodes such that they could belong to more than one group simultaneously, but where all group costs are equal and all node capacities are equal; the second and third sets of simulations consider the case where group costs and node capacities respectively are heterogeneous. For each set of experimental conditions, ten simulation runs were carried out, each consisting of $5 \times 10^{6}$ time-steps.

\subsection{Exclusive groups}

We begin by describing the base system behaviour when group membership is exclusive: that is, all individuals have a TAE capacity of one and all groups have a TAE cost of one. Therefore, any individual initiating or joining a new group must first leave its current group. This corresponds to the situation found in many opinion 


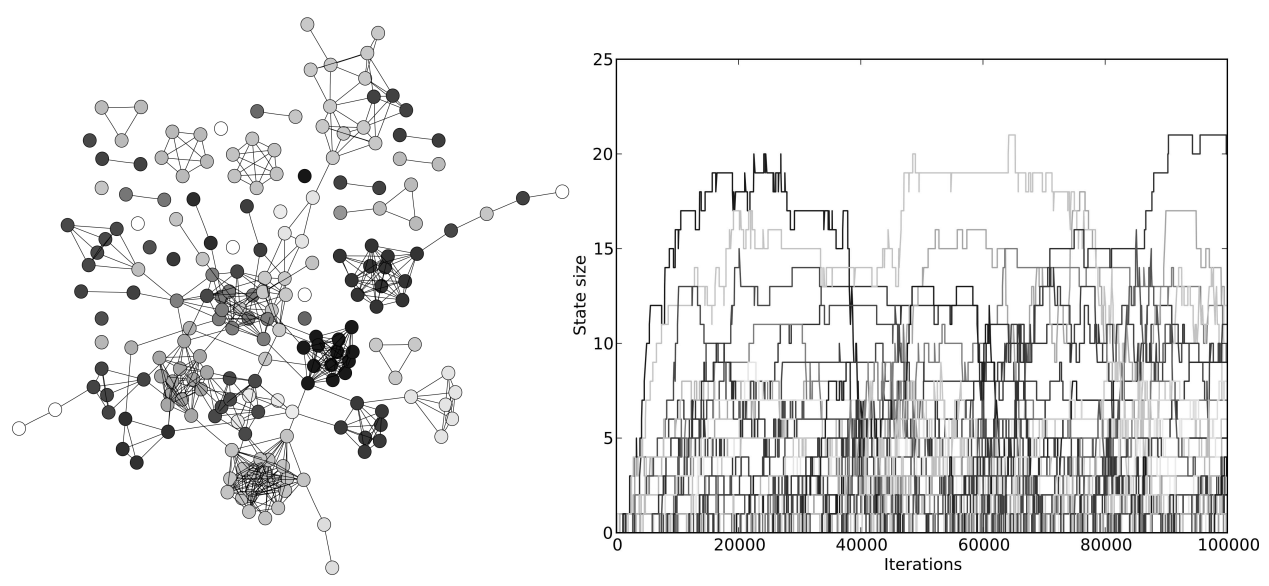

Fig. 1. An example network structure and group dynamic with exclusive group membership.

dynamics models (although the exact mechanisms used to modify node state and edge location may differ from model to model). Figure 1 shows the final network structure (with nodes coloured by group affiliation) and evolution through time of group sizes.

Under these circumstances, the population evolves to what may be termed a connected community structure. There is a strong overlap between the set of nodes that share the same topological community and the set of nodes that share the same group affiliation. However, the presence of a low level of random rewiring prevents these clusters from disconnecting entirely, and allows the continued propagation of group affiliation between communities. This continuing connectivity, combined with the occasional initiation of novel groups maintains the population in a state of dynamic equilibria: aggregate network level properties of the network stabilise, while individual nodes continue to update their affiliation and neighbourhood. The group size plot in Figure 1 illustrates the dynamic nature of groups: many groups are initiated, some survive to grow and assume a dominant position in the population, but even these are eventually overtaken and replaced.

\subsection{Series A: Increasing individual TAE capacity}

The first set of simulations was used to investigate the effect of increasing the populations TAE capacity, such that individuals could belong to multiple groups simultaneously. Within a single simulation, all nodes had the same TAE capacity (in the range $[1,5]$ ) and all groups had a TAE cost of one. Two social network properties were measured: Modularity quantifies the extent to which the social network exhibits community structure [31]. The measured values represent the optimal partition identified by a community detection algorithm. A random network typically displays a modularity value of approximately 0.3 , and values above this indicate the 

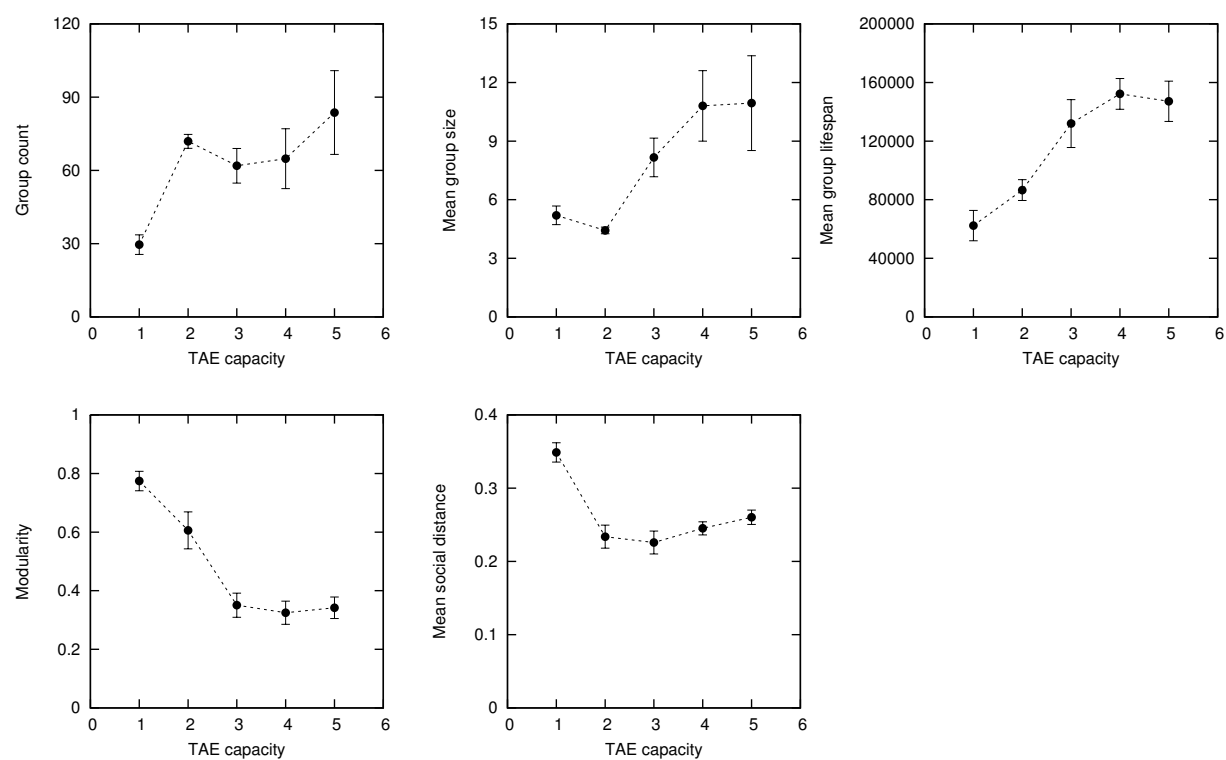

Fig. 2. Series A: Effects of varying TAE capacity. All groups have a TAE cost of 1 . Other parameters as described in text. Error bars show \pm 1 SD.

presence of significant community structure. Mean social distance (MSD) quantifies the level of observed homophily in the population. MSD is calculated as the mean of the social distances between neighbouring individuals (i.e., along each edge of the social network). When individuals are randomly distributed in a population, MSD is approximately 0.33 ; values below this indicate that more edges are between individuals who are close in sociodemographic space than would be expected in a random population. Three group level properties were measured: Group count is the number of live groups (i.e., containing at least two individuals) at the end of the simulation run. Mean group size is the mean size of live groups at the end of the simulation run. Mean group lifespan is the mean age of all groups that have existed at any time during the simulation run.

The simulation results indicate that increasing TAE capacity above one has significant effects on network structure, observed homophily and group dynamics (Figure 2). The level of community structure decreases until it is comparable to that of a random network of equivalent size and density [16]. However, while social ties are distributed more homogeneously throughout the population for TAE capacity above two, they are more likely to be between similar individuals. The combination of these two trends suggests that as individuals belong to more groups, they are less likely to become disconnected from the population, but have more opportunity to leave groups containing individuals dissimilar to themselves. Unexpectedly, although the 

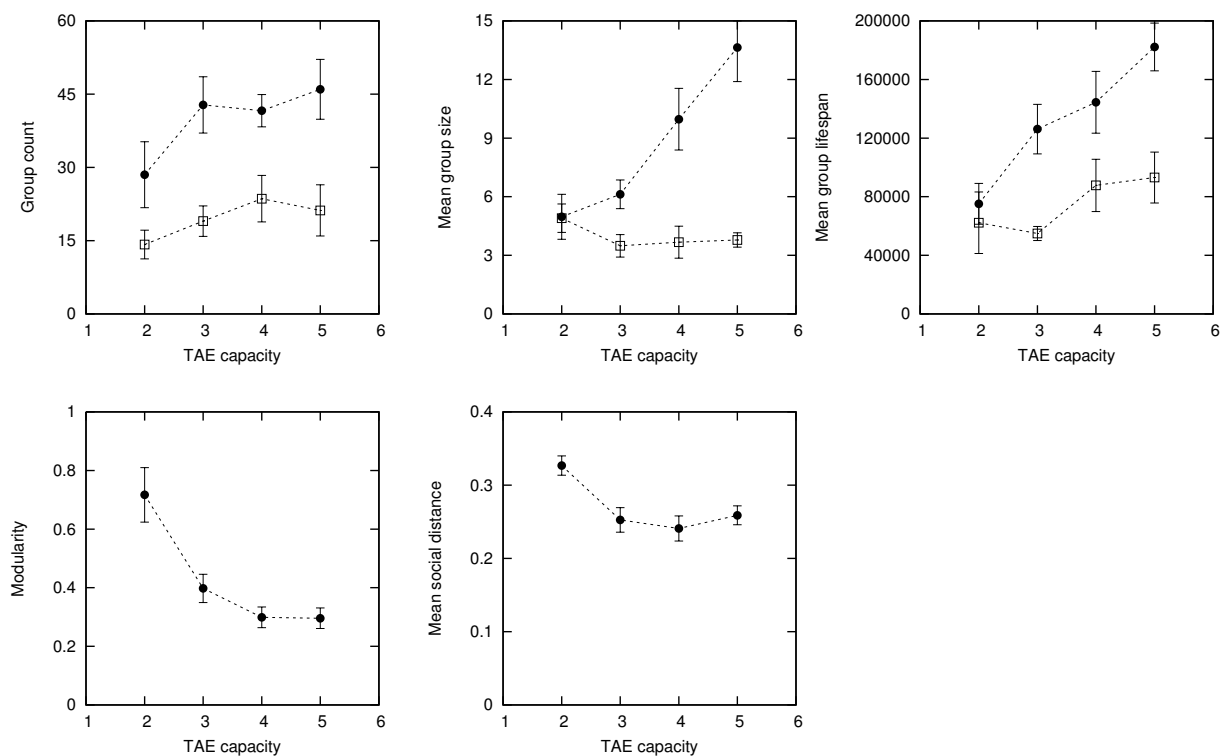

Fig. 3. Series B: Effects of heterogeneous group TAE costs. Each group has an equal change of having a TAE cost of one (solid circles) or two (hollow squares). Other parameters as described in text. Error bars show $\pm 1 \mathrm{SD}$. Note that TAE capacity ranges from two to five, and group count ranges from zero to sixty; otherwise, axes are identical to corresponding plots in Figure 2.

theoretical limit on the number of possible groups increases linearly with increasing TAE capacity, the number of groups observed increases more slowly. After an initial jump from 29.6 to 71.9 groups as TAE capacity is increased from one to two, the mean group count remains relatively constant between 61.9 and 83.7 as TAE capacity is further increased to five. Similarly, the mean size and lifespan of observed groups increases non-linearly with increasing TAE capacity. Two interesting points emerge. At higher capacities, the variance in group size increases. rather than a collection of roughly equal-sized groups, populations tend to consist of a few larger groups and many smaller groups. Also, the similarity between the shape of these two trends suggests that lifespan may be related to group size.

\subsection{Series B: Heterogeneous group TAE costs}

A second set of simulations was used to investigate the effect of heterogeneous group TAE costs, such that some groups made greater demands on the time and energy of their members than others. In these simulations, each time a new group was initiated, it had an equal chance of having a TAE cost of one or two. Properties measured were as above; however, those pertaining to groups were measured separately for each class of groups.

The simulation results indicate that, as may be anticipated, less costly groups 

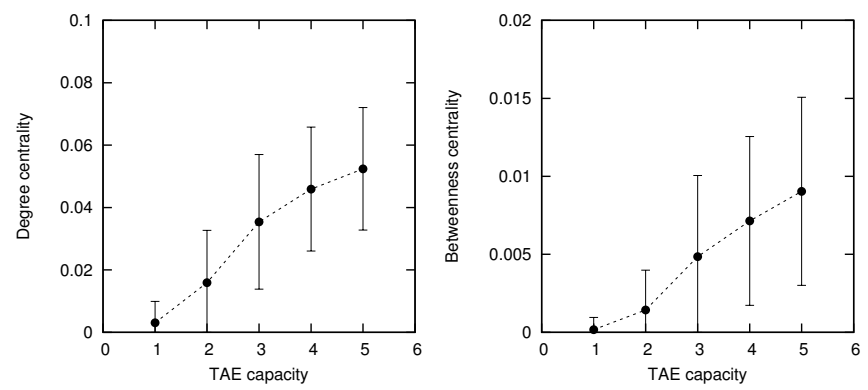

Fig. 4. Series C: Effects of heterogeneous TAE capacity. Each individual has an equal chance of having a TAE capacity between 1 and 5 . All groups have a TAE cost of one. Other parameters as described in text. Error bars show $\pm 1 \mathrm{SD}$.

are maintained in the population in greater quantities than more costly groups (Figure 3). Similarly, the less costly groups are larger, and survive longer. One less expected observation is that the mean size of the more costly groups remains constant as TAE capacity increases, while that of the less costly groups grows rapidly (more rapidly, in fact, than was the case when all groups were of equal cost).

\subsection{Series C: Heterogeneous individual TAE capacities}

A final set of simulations was used to investigate the effect of heterogeneous individual TAE capacities, such that some individuals have more time and energy to allocate to group activities than others. In these simulations, each agent in a population had a TAE capacity drawn uniformly at random from the range [1,5]. In these simulations, all groups had a TAE cost of one. For these simulations we also measured node-level properties, including the degree centrality (normalised number of neighbours each node has) and betweenness centrality (normalised number of shortest paths that each node lies on).

The simulation results indicate that, as anticipated, individuals with greater TAE capacity, while initially distributed at random throughout the population, came to occupy more central locations in the social network (Figure 4). Otherwise, there were minimal discernible differences in the population and group-level behaviour.

\section{Discussion}

Patterns of group affiliation and the structure of social ties in a population interact in a non-trivial fashion. In this paper we have explored the effect of varying levels of competition on the dynamics of group affiliation using a simple coevolutionary model. By allowing individual nodes to belong to multiple groups, we have moved 
beyond the type of exclusive group affiliation associated with existing opinion dynamics and cultural evolution models. By altering the capacity of individuals to belong to multiple groups, and the costs associated with group membership, we have been able to explore interactions between social network evolution and group affiliation dynamics under different competitive conditions. In this section, we summarise our observations of the NEG model behaviour and relate them to back to our theoretical concerns. We then describe a possible domain for a more concrete instantiation of the model: the social networks connecting university students, and their affiliations with clubs and societies. Evaluating the current model from this perspective, we suggest several ways in which the assumptions and design could be refined in future model designs.

Our observations may be summarised as follows:

- Reducing the level of competition between groups (Series A) reduces the level of community structure observed in the social network and increases the level of observed homophily. These changes may be related by the fact that decreasing community structure increases the possibility of an individual being exposed to groups to which they are more closely aligned in sociodemographic space.

- A greater number of larger and more long-lived groups are sustained by populations when competition is decreased (Series A). Most of these gains occur with the initial removal of exclusive membership and further reductions in competition have less impact on group properties.

- When groups have different costs of affiliation (Series B), less costly groups are maintained in greater numbers than more costly groups. When the level of competition is decreased, less costly groups experience more dramatic gains in number, size and lifespan than more costly groups.

- When individuals have different capacities for group affiliation (Series C), individuals with greater capacity come to occupy more central locations in the social network.

What then are the implications of non-exclusive groups for social structure and societal integration? In comparison to the scenario in which all groups are exclusive, allowing multiple affiliations results in a more complex network structure that is subject to conflicting forces. As the level of competition is decreased, the population is less inclined to fragment into disconnected communities and network structure becomes more topologically integrated. At the same time, the level of observed homophily increases, suggesting that the greater range of available options for association has actually reduced the level of sociodemographic integration.

To make some of the ideas discussed in this paper more concrete, we evaluate how the mechanisms embodied in the NEG model might play out in a real domain. Consider the example of student society memberships in a typical university. Students at a university have the opportunity to join and participate in clubs and societies focused around shared recreational, political or social interests. They learn about the existence of particular societies both through their social contacts and 
via general advertising. Membership in societies involves attendance at meetings or other society activities, which entail a time cost, and which may act as venues for the formation of new social contacts. The time and energy costs of being involved in societies are likely to vary among societies, and the amount of time individuals are willing to devote to participation in society activities (as opposed to, say, studying) is likely to vary among students. Over time, the patterns of membership across societies will change, as some societies flourish and grow, others fade away and new societies are founded to address hitherto unmet needs. Is it possible to characterise the organisational and structural attributes of societies and predict which are likely to succeed? Similarly, the patterns of social interaction between students will evolve over time [37, 29]. Students vary in terms of their sex, age, ethnicity, academic program and other variables. What type of social network structures emerge? Are these structures integrated or segregated? To what extent (if any) can this structure be explained in terms of patterns of society co-memberships?

On the basis of the results reported here, we may predict that less costly groups may find it easier to thrive, but that more costly groups may retain more diversity. However, viewing the NEG model through the lens of the above example reveals several limitations of the current set of assumptions. We discuss here four specific areas that warrant further attention: individual utility, group efficacy, group identity and group structure. Regarding the first two areas, the current model omitted an explicit description of both an individual's utility function and a group's efficacy. These are clearly important and related, and will have a bearing on system dynamics. Students deciding whether or not to join or leave a particular society will need to weigh up the benefits of membership against the costs incurred (including the foregone opportunities to join other groups). A student's assessment of membership benefits is likely to be influenced by the extent to which a group is achieving its goals. In turn, group success is likely to be a more complex function of membership and context than currently considered. Real groups must balance the cohesion provided by intra-group ties with the access to resources and information provided by inter-group ties [30]. Furthermore, the implications of groups with a finite, or timelimited, goal remain to be explored. A richer definition of individual utility could also enable a more principled approach to the initiation of new groups. Rather than occurring at random, new societies are founded in response to the existence of needs that are not met by currently existing societies.

The third potential area of model development is the definition of group identity. In reality, not all groups are interchangeable, and the exclusiveness of groups may apply only to other groups of the same type. For example, a student belonging to a society representing a particular political party may be discouraged from joining societies representing competing political parties, but not from joining societies formed around sporting or musical interests (as in [8]). The final area is group structure. While the NEG model does implicitly include group structure in terms of the network of social ties between group members, it is interesting to consider the implications this structure may have for group efficacy on the one hand and 
individual utility on the other: Do some network structures make for more successful groups than others? To what extent might an individual's utility function be tied to their status within a group?

It is apparent that there is much complexity present even in the relatively circumscribed domain of student societies. Other domains will impose further sets of constraints on model definition. Much remains to be done in order to better understand the relationship between social networks and group dynamics more generally. Modelling competitive dynamics of non-exclusive groups represents an important step forward and we believe our approach has considerable potential for future development.

\section{Acknowledgements}

We thank two anonymous reviewers for their helpful comments - and specifically for suggesting the example domain described in Section 7 - and acknowledge financial support from the EPSRC (EP/D00232X/1).

\section{References}

[1] Amblard, F. and Jager, W., Emergent social networks resulting from different processes of interaction, in The Fifth Conference of the European Social Simulation Association, Brescia, Italy (2008).

[2] Axelrod, R., The dissemination of culture: A model with local convergence and global polarization, The Journal of Conflict Resolution 41 (1997) 203-226.

[3] Babchuk, N. and Edwards, J. N., Voluntary associations and the integration hypothesis, Sociological Inquiry 35 (1965) 149-162.

[4] Borgatti, S. P., Mehra, A., Brass, D. J., and Labianca, G., Network analysis in the social sciences, Science 323 (2009) 892-895.

[5] Bryden, J., Funk, S., Geard, N., Bullock, S., and Jansen, V. A. A., Theseus's ship: stable community structure in dynamic networks (2010), in preparation.

[6] Castellano, C., Fortunato, S., and Loreto, V., Statistical physics of social dynamics, Reviews of Modern Physics 81 (2009) 591-646.

[7] Centola, D., Gonzalez-Avella, J. C., Eguiluz, V. M., and Miguel, M. S., Homophily, cultural drift, and the Co-Evolution of cultural groups, Journal of Conflict Resolution 51 (2007) 905-929.

[8] Chattoe, E., Using simulation to develop testable functionalist explanations: a case study of church survival, The British Journal of Sociology 57 (2006) 379-397.

[9] Cummings, J. N., Work groups, structural diversity, and knowledge sharing in a global organization, Management Science 50 (2004) 352-364.

[10] Davis, K., Human Society (McMillan \& Co. Ltd., New York, 1965).

[11] Deffuant, G., Neau, D., Amblard, F., and Weisbuch, G., Mixing beliefs among interacting agents, Advances in Complex Systems 3 (2001) 87-98.

[12] Edmonds, B., Norling, E., and Hales, D., Towards the evolution of social structure, Computational \& Mathematical Organization Theory 15 (2009) 78-94.

[13] Feld, S. L., The focused organization of social ties, The American Journal of Sociology 86 (1981) 1015-1035.

[14] Gilbert, N. and Troitzsch, K. G., Simulation for the Social Scientist (Open University Press, Buckingham, 1999). 
[15] Gould, R. V., Collective action and network structure, American Sociological Review 58 (1993) 182-196.

[16] Guimerà, R., Sales-Pardo, M., and Amaral, L. A. N., Modularity from fluctuations in random graphs and complex networks, Phys Rev E $\mathbf{7 0}$ (2004) 025101.

[17] Hegselmann, R. and Krause, U., Opinion dynamics and bounded confidence models, analysis, and simulation, Journal of Artificial Societies and Social Simulation $\mathbf{5}$ (2002).

[18] Holme, P. and Newman, M. E. J., Nonequilibrium phase transition in the coevolution of networks and opinions., Physical Review E $\mathbf{7 4}$ (2006) 056108.

[19] Huet, S., Deffuant, G., and Jager, W., A rejection mechanism in 2D bounded confidence provides more conformity, Advances in Complex Systems 11 (2008) 529-549.

[20] Jackson, M. O. and Roger, B. W., Meeting strangers and friends of friends: How random are social networks?, The American Economic Review 97 (2007) 890-915.

[21] Kimura, D. and Hayakawa, Y., Coevolutionary networks with homophily and heterophily., Phys Rev E Stat Nonlin Soft Matter Phys 78 (2008) 016103.

[22] Kozma, B. and Barrat, A., Consensus formation on adaptive networks, Physical Review E 77 (2008) 016102.

[23] MacIver, R. M. and Page, C. H., Society: An Introductory Analysis (McMillan \& Co. Ltd., London, 1952).

[24] McAdam, D. and Paulsen, R., Specifying the relationship between social ties and activism, The American Journal of Sociology 99 (1993) 640-667.

[25] McPherson, J. M. and Rotolo, T., Testing a dynamic model of social composition: Diversity and change in voluntary groups, American Sociological Review 61 (1996) 179-202.

[26] McPherson, J. M., Smith-Lovin, L., and Cook, J., Birds of a feather: homophily in social networks, Annual Review of Sociology 27 (2001) 415-444.

[27] McPherson, M., An ecology of affiliation, American Sociological Review 48 (1983) $519-532$.

[28] McPherson, M., A Blau space primer: prolegomenon to an ecology of affiliation, Industrial and Corporate Change 13 (2004) 263-280.

[29] Moody, J., Race, school integration, and friendship segregation in America, American Journal of Sociology 107 (2001) 679-716.

[30] Newman, L. and Dale, A., Homophily and agency: Creating effective sustainable development networks, Environment, Development and Sustainability 9 (2007) 79 90 .

[31] Newman, M. E. J., Modularity and community structure in networks., Proc Natl Acad Sci U S A 103 (2006) 8577-8582.

[32] Olson, M., The Logic of Collective Action: Public Goods and the Theory of Groups (Harvard University Press, 1974).

[33] Pescosolido, B. A. and Rubin, B. A., The web of group affiliations revisited: Social life, postmodernism, and sociology, American Sociological Review 65 (2000) 52-76.

[34] Popielarz, P. A. and McPherson, J. M., On the edge or in between: Niche position, niche overlap, and the duration of voluntary association memberships, The American Journal of Sociology 101 (1995) 698-720.

[35] Putnam, R., Bowling Alone: The Collapse and Revival of American Community (Simon \& Schuster Ltd, 2001).

[36] Sandell, R., Organizational life aboard the moving bandwagons: A network analysis of dropouts from a swedish temperance organization, 1896-1937, Acta Sociologica 42 (1999) 3-15.

[37] Shrum, W., Cheek, Jr., N. H., and MacD. Hunter, S., Friendship in school: Gender 
and racial homophily, Sociology of Education 61 (1988) 227-239.

[38] Simmel, G., Conflict and the Web of Group Affiliations (Free Press, New York, 1955), translated by K. H. Wolff and R. Bendix.

[39] Snow, D. A., Zurcher, L. A., and Ekland-Olson, S., Social networks and social movements: A microstructural approach to differential recruitment, American Sociological Review 45 (1980) 787-801.

[40] Southern Poverty Law Center (SPLC), The second wave: Return of the militias (2009), report available at http://www.splcenter.org/.

[41] Stern, C., The evolution of social-movement organizations: Niche competition in social space, European Sociological Review 15 (1999) 91-105.

[42] Watts, D. J., Dodds, P., and Newman, M. E. J., Identity and search in social networks, Science 296 (2002) 1302-1305.

[43] Yang, G., Environmental NGOs and institutional dynamics in China, The China Quarterly 13 (2005) 113-130. 